\title{
Eine unabhängige Judikative als Gegengewicht zur Erosion europäischer Strafrechtsprinzipien? ${ }^{1}$
}

\section{A. Das verspielte Erbe: Europäische Strafrechtsprinzipien in Erosion}

Am Anfang der Entwicklung europäischer Strafrechte standen - jedenfalls der Idee nach - Freiheit, Menschenwürde und universale Menschenrechte. Das sind die zentralen Merkmale des Gesellschaftsvertrages als gedanklicher Fixpunkt der Aufklärung. Das Strafrecht ist kein Allheilmittel staatlicher Sicherheitsinteressen, sondern schützt die Rechtsunterworfenen vor Zugriffen auf den Kernbereich von Freiheit und Menschenwürde - im Staat und darüber hinaus. Strafrechtsprinzipien sind dabei Schutzformen der Freiheit, die in das Strafrecht übersetzt werden müssen. ${ }^{2}$ Jene Schutzformen werden in Prozessen heftiger Erosion verschlissen.

\section{Das Leitprinzip: Strafgesetzlichkeit}

Die Strafgesetzlichkeit ist das zentrale Prinzip des Strafrechts. Mit der Strafgesetzlichkeit beginnt das legitime, die Macht des Staates beschränkende Strafrecht. »Keine Strafe ohne Gesetz« - so lautet die Kurzformel der Strafgesetzlichkeit. Sie stellt die fundamentalste Errungenschaft eines aufgeklärten, modernen und rechtsstaatlichen Strafrechts dar. Die Botschaft der Strafgesetzlichkeit liegt in der Machtbegrenzung und im Schutz der persönlichen Freiheit vor dem strafenden Staat. ${ }^{3}$ Gesellschaftsvertrag und Strafgesetzlichkeit sind untrennbar miteinander verbunden. Beides konstituiert den freiheitlichen demokratischen Rechtsstaat. Sie ist Ausfluss rechtsstaatlicher Grundsätze und kann als notwendige Konsequenz von Demokratie und Gewaltenteilung begriffen werden.

Fragt man neben der formalen Bedeutung, also der parlamentarischen Strafgesetzlichkeit, nach der inhaltlichen Bedeutung, so folgen hieraus Begrenzungen für den Staat. Dieser kann nicht einfach alles, was gegen Regeln oder Interessen verstößt, was störend oder gefährlich sein kann, als strafwürdig ansehen. Ein solch ausuferndes Strafrecht ist eine Bedrohung für die Freiheit. Strafrecht soll nach der Grundlegung der Aufklärungsphilosophien nur dann Anwendung finden, wenn die Freiheit eines Bürgers durch einen anderen in ihrem Kern verletzt wird. Dies macht den speziellen Charakter des vom Strafrecht erfassten Unrechts aus. Die Zügel für die staatliche

1 Ergänzte Fassung eines Vortrages, der sowohl in der Universität von Luxemburg anlässlich des Symposiums »Quel contrôle juridictionnel dans l'espace pénal européen?« am 9. November 2007 und in abgewandelter Form im Unterausschuss Europarecht des Rechtausschusses des Deutschen Bundestages anlässlich eines öffentlichen Expertengesprächs am 28. November 2007 gehalten wurde.

2 Vgl. hierzu und auch der im folgenden angesprochenen Strafrechtsprinzipien Albrecht, Die vergessene Freiheit, 2. Auflage, 2006, 53ff. m.w.N.

3 Vgl. hierzu weiterführend die Ansätze von Naucke in Gesetzlichkeit und Kriminalpolitik, 1999 und Über die Zerbrechlichkeit des rechtsstaatlichen Strafrechts, 2000. 
Machtbegrenzung sind die bekannten Trassen des Gebotes der Bestimmtheit strafrechtlicher Normen, des Analogie- und Gewohnheitsrechtsverbotes, des Rückwirkungsverbotes, alles erfahrene und erlebte Werte europäischer Rechtstradition.

Weder in nationaler noch europäischer Gesetzgebung und Rechtsanwendung genießt dieses Prinzip besonders tief greifenden Respekt. So schafft der Gesetzgeber Tatbestände, die selbst für Eingeweihte nicht mehr verständlich sind. Tatbestände, bei denen es kaum mehr möglich erscheint, das strafbare Verhalten von einem alläglichen Normalverhalten zu unterscheiden. Der Straftatbestand der Geldwäsche (§ $261 \mathrm{StGB})$ ist ein solcher Tatbestand. Sein Wortlaut ist einerseits kompliziert, andererseits kennt er kaum noch eigene Zurechnungskriterien. So geht es den meisten Tatbeständen, die über das Instrument der europäischen Richtlinie geboren oder zumindest gefördert werden. Das moderne Strafrecht wird von einer Fülle unbestimmter Rechtsbegriffe gekennzeichnet. Statt eines Verletzungserfolges genügt oftmals nur noch die abstrakte Gefahr einer Verletzung, um das Strafrecht bemühen zu können. So genannte Gefährdungsdelikte - im Straßenverkehr, im Umweltstrafrecht, im Wirtschaftsstrafrecht werden von der Ausnahme, die sie ursprünglich waren, zur Regel. Im Ergebnis führt dies zu einer Vorverlagerung von Strafbarkeit, damit auch zu einer Entgrenzung der Macht, die mit dem Strafrecht unweigerlich verbunden ist. Dann ist die Demokratie nur noch eine leere Hülle, in der die Exekutive das Strafrecht an sich reißen und sich die Strafgesetzlichkeit zur Beute machen kann. ${ }^{4}$

\section{Zentrale Prinzipien europäischer Aufklärung}

\section{Schuldprinzip}

Die Strafgesetzlichkeit ist Ausgang und Anfang des europäischen Aufklärungserbes. Das Schuldprinzip erweitert unsere Überlegungen und hat eine wichtige Funktion: Es ist die Begrenzung der staatlichen Strafmacht. Aber wie ist die normative Realität des individuellen Schuldkonstrukts in europäischer Perspektive aufgestellt? Nicht mehr personelle Zurechnung wird als primärer Maßstab des Schuldverständnisses in einem werdenden europäischen Strafrecht angesehen. Vielmehr geht es den Verfassern bestimmter Entwürfe gerade um die Abkopplung von individueller Verantwortung. Beabsichtigt ist die flächendeckende Bewältigung von Risiken der »modernen «Industriegesellschaften: Aggressive Durchsetzung von Partikularinteressen in Wirtschaft und Umwelt, weltgesellschaftliche Wohlstandsdiskrepanzen, sozio-ökonomische und politische Konfliktlagen, unkalkulierbare Risiken einer ökonomisch angetriebenen Wissenschafts- und Technikentwicklung etc. Staatliche Reaktionen dagegen sind gefordert, aber nicht um den Preis der Aufgabe des Prinzips individueller Verantwortlichkeit.

Die Folgenorientierung des Strafrechts wechselt vom individuellen Täter hin zu gesellschaftlichen Problemlagen - primär symbolisch. Mit der Entwicklung rein symbo-

4 Die Formulierung ist angelehnt an den eindrucksvollen Titel einer kritischen Analyse des Präsidenten des OLG Brandenburg a.D. Peter Macke, Die Dritte Gewalt als Beute der Exekutive, in: DRiZ 1999, 481ff. 
lischer Rechtsfunktionen als Erzeugung von Sinnbildern und scheinbaren Lösungsmustern gegenüber realen gesellschaftlichen Problemlagen werden die Eingriffe in rechtsstaatliche Grundprinzipien noch bedrohlicher. Soll in Zukunft schon leichte, das heißt unbewusste Fahrlässigkeit, Kriminalstrafe nach sich ziehen (zum Beispiel beim Betrug $^{5}$ ), fehlt jede Anknüpfung an individuelle Vorwerfbarkeit. Kriminalisiert werden nur noch Risikolagen, das heißt der Systemschutz und nicht mehr die persönliche Verantwortlichkeit steht im Fokus der Aufmerksamkeit des Strafrechts. Schuld als strafrechtliche Anknüpfungskategorie scheint in den bisherigen Entwürfen europäischer Strafrechtsentwicklung nahezu verloren.

\section{Verhältnismäßigkeitsprinzip, Legalitätsprinzip, Offizialprinzip und fair trial}

Wurzeln europäischer Aufklärung sind darüber hinaus das Verhältnismäßigkeitsprinzip als rechtliche Schranke staatlicher Gewaltanwendung und das Legalitätsprinzip als Willkürschranke für staatliche Rechtsanwendung. Ferner das Offizialprinzip als Garant des öffentlichen Strafrechts, welches im Rechtsstaat nie Privatsache sein darf, sondern der Wahrheits- und Unrechtsfeststellung des öffentlichen Strafrechts - einer Kernfunktion des demokratischen Rechtsstaats - dient. Schließlich bilden die Prinzipien des fairen Strafverfahrens das Fundament des Strafprozesses im freiheitlichen Rechtsstaat. Fairness im Strafverfahren ist das Fundament aller Grundsätze des rechtsstaatlichen Strafprozesses. Nicht von ungefähr wird der Strafprozess als Seismograph freiheitlicher Verfasstheit einer Gesellschaft angesehen. Fairness ist eine gewollt kostspielige Ressource, die - nicht selten mit äußerster Anstrengung für die Strafjustiz - die freiheitliche Verfasstheit eines Kriminaljustizsystems repräsentiert. Es ist ein Kennzeichen aktueller Kriminalpolitik, in Verkennung dieser Ausgangslage durch Kosten und Mühen sparende Eingriffe in das Verfahrensrecht und die Organisationsstruktur des Kriminaljustizsystems den Richtern Entlastung zu versprechen. Beispiele sind die stets von der Kriminalpolitik geforderte Rücknahme von Beweisantragsrechten und die damit erzeugte Einschränkung richterlicher Erkenntnis, was sich auch im Trend zur permanenten Verkleinerung von Spruchkörpern und dem relativen Rückgang an Richterstellen zeigt. ${ }^{6}$

Der Abbau rechtsstaatlicher Sicherungen wird in der kriminalpolitischen Diskussion mit Gefahren legitimiert, die dem rechtstreuen Bürger angeblich im Übermaß drohen. Im Vordergrund steht das kriminalpolitisch gepflegte Szenarium einer »explodierenden Gewaltkriminalität«, insbesondere der so genannte Terrorismus. ${ }^{7}$ Mit zugespitzten Irrationalitäten verschärft, verformt und zerstört eine populistische Kriminalpolitik die rechtsstaatlichen Grundlagen des Kriminaljustizsystems. Die Bürger akzeptieren diese Erosionen von Freiheitsrechten, weil vermeintlich krimineller Notstand besteht. Sie selbst rufen nach einer Sicherheitsgesellschaft, ${ }^{8}$ ohne Bewusstsein für die katastropha-

5 Art. 2 Corpus Juris Florence.

6 Stellungnahme der NRV vom 31.01.2008 zu ausgewählten Fragen des »Questionnaire for the 2008 CCJE opinion concerning the quality of judicial decisions «, 1ff., http://www.nrvnet.de/downloads_stellung/60.pdf.

7 Albrecht, Kriminologie - Eine Grundlegung zum Strafrecht, 3. Auflage, 2005, 352ff.

8 Singelnstein/Stolle, Die Sicherheitsgesellschaft, 2006, 87ff. 
len Folgen ihres Rechtsverlustes. Abgesehen davon, dass es diesen Notstand nicht gibt, wofür eine autonome Kriminologie Aufklärungsdienste leistet, ${ }^{9}$ müsste verantwortliche Politik eines deutlich machen: Die Aufgabe des Prinzips der Freiheit in einem funktionierenden Kriminaljustizsystem führt nicht zu mehr öffentlicher Sicherheit, sondern zu deren Vernichtung.

\section{B. Das Mängelprofil der Dritten Gewalt auf nationaler und supranationaler Ebene}

Richterliche Unabhängigkeit und Unparteilichkeit sind essentials des Prinzips der Gewaltenteilung, demokratischer Rechtsstaatlichkeit und damit Grundpfeiler eines europäischen Rechtsverständnisses. Vermag die Unabhängigkeit einer Dritten Gewalt dem Verfallsprozess rechtsstaatlicher europäischer Strafrechtsprinzipien den notwendigen Einhalt zu geben? Wir wollen dieser Frage in zwei Schritten nachgehen. Die Auswirkungen der Dominanz der Exekutive lassen sich im nationalen Recht bereits deutlich kennzeichnen (I.), was im supranationalen Kontext noch gar nicht in diesem Umfang feststellbar sein kann. Die Gefährdung richterlicher Unabhängigkeit im Bereich der EU zeigt sich dort jedoch ähnlich gravierend, wenn auch in anderen Bereichen (II.).

I. Die Dominanz der Exekutive und die Randständigkeit der Dritten Gewalt am Beispiel des deutschen Kriminaljustizsystems

Die verfassungsrechtlichen Ableitungen der materiell-rechtlichen Prinzipien erfahren erst in der Unabhängigkeit der Gerichte ihre wahre Entfaltung. Aber wie steht es rechtstatsächlich mit der Unabhängigkeit der Dritten Gewalt? Folgendes Mängelprofil, das der richterlichen Unabhängigkeit die rechtsstaatliche Luft abschnürt, dürfte in weiten Zügen europaweit verallgemeinerbar sein:

- starke Zunahme der Fallbelastung bei Abnahme personeller Ressourcen in der Gerichtsbarkeit,

- starker exekutivischer Druck auf einen bereits so genannten »ökonomisierten Richter ${ }^{10}$,

- starker Zwang zur informellen Verständigung und normativen Konsensorientierung bei gleichzeitiger Zunahme an Geheimhaltung des ehemals offenen Strafprozesses schlägt sich in einer Informalisierung der Dritten Gewalt nieder, was deutlicher als Rechtsauflösung beschreibbar ist.

1. Informalisierung des Strafverfahrens und exekutivisches Recht

Die Vielzahl der Verdachtsfälle in Bagatell- und Jugendstrafverfahren, die ressourcenverzehrende Verstrickung in zahlreiche Umfangverfahren des Wirtschaftsstrafrechts, unzeitgemäße und fremdbestimmte Organisationsformen des Kriminaljustizsystems einschließlich permanenter Ressourcenverknappung und nicht zuletzt der Handlungs-

9 Albrecht, Kriminologie - Eine Grundlegung zum Strafrecht, 3. Auflage, 2005, 337ff. (Kriminalpolitische Bedrohungsszenarien: »Ausländerkriminalität«, »Organisierte Kriminalität «, »Terrorismus «).

10 Schütz, Der ökonomisierte Richter, 2005. 
druck, den eine angsterzeugende populistische Kriminalpolitik entfacht, erzwingen für die Kriminaljustiz verfahrensökonomische Erledigungsstrategien. ${ }^{11}$ Trotz zunehmender Fallast soll die Justizorganisation funktionsfähig bleiben. Anstelle angezeigter normativer Entkriminalisierung werden Informalisierungen des Strafverfahrens geschaffen. Jeder zweite anklagefähige Fall wird heute in Deutschland - zumeist ohne Einbeziehung der Richter - von der Staatsanwaltschaft eingestellt. ${ }^{12}$

Mit dieser Informalisierung beginnt keine Erfolgsgeschichte des Strafrechts. Verfahrenseinstellungen haben kontinuierlich prozentual zugenommen. Damit war eine Vermeidung belastender Verfahren nicht herbeigeführt worden. Verändert hatte sich lediglich die Form der einst kritisierten Stigmatisierung: Der Richter wurde durch den Staatsanwalt ersetzt. Um den Durchsetzungs- und damit Geltungsproblemen des Strafrechts zu entgehen, darf die Geltung der Norm verfahrenstechnisch außer Kraft gesetzt werden. Auf die Stoffüberlastung reagiert das Kriminaljustizsystem mit Mechanismen der Stoffbegrenzung. Opportunität und Absprachen im Strafverfahren sind die Mittel dieser Stoffbegrenzung. Diese Art der Erledigung wurzelt nicht mehr in wohlfahrtsstaatlichen Rechtskonzeptionen, wie sie die kritische Kriminologie einstmals selbst verfolgte, sondern in Überlegungen administrativer Rationalisierung. ${ }^{13}$ Das dabei entstehende exekutivische Recht impliziert einen gehaltlosen Rechtsbegriff. ${ }^{14}$ Die einst durch die Verpflichtung auf die individuelle Freiheit im Recht sich legitimierenden Gesetze sind nur noch poröse Sammelsurien bürokratischer Praktiken im zweifelhaften Dienst des Interventionsstaats, dem das Attribut »sozial « in weiten Bereichen abhanden gekommen ist. Es besteht insgesamt die Gefahr, dass die Unabhängigkeit und die konstitutiven Prinzipien der Dritten Gewalt durch ein exekutivisches Konzept informalisierender Verfahrensbewältigung ausgehöhlt werden. ${ }^{15}$

\section{Zweifelhafte Modernisierung: Ökonomisierung der Justiz}

Ein Phänomen, welches den gesamten Gerichtssektor betrifft, ist das der »Verbetriebswissenschaftlichung ${ }^{16}$. Gemeint ist die Übertragung von Methoden der freien Wirt-

11 Siehe hierzu den Sonderforschungsbereich 227 der Deutschen Forschungsgemeinschaft (1. Forschungsphase »Staatsanwaltschaftliche Diversion«) Albrecht (Hrsg.), Informalisierung des Rechts, 1990.

12 Albrecht, Kriminologie - Eine Grundlegung zum Strafrecht, 3. Auflage, 2005, $188 \mathrm{ff}$.

13 Siehe hierzu den Abschlussbericht des Sonderforschungsbereichs 227 der DFG (2. Forschungsphase »Gerichtsverfahren und Diversion«) Ludwig-Mayerhofer, Das Strafrecht und seine administrative Rationalisierung. Kritik der informalen Justiz, 1998.

14 Naucke, Gesetzlichkeit und Kriminalpolitik, 1999, 196ff., 256ff.

15 Vgl. hierzu auch Hamm, Ist die Entformalisierung des Strafrechts und Strafprozesses unaufhaltsam?, in: Jenseits des rechtsstaatlichen Strafrechts, Institut für Kriminalwissenschaften und Rechtsphilosophie, Frankfurt a.M., 2007, 521ff.

16 Ganz ähnliche Prozesse einer verfehlten Ökonomisierung finden wir in der auf Privatisierung abstellenden Wissenschaftspolitik in der Bundesrepublik Deutschland (vgl. hierzu die Beiträge in KritV 3/2007, Hochschullehrerversammlung des Fachbereichs Rechtswissenschaft der Johann Wolfgang Goethe-Universität, Sorge um die Wissenschaftsfreiheit und die Qualität universitärer Forschung und Lehre, 228ff.; Augsberg, Selbstreferenz als Gesetzgebungsprogramm, 236ff.) . 
schaft zur Steigerung von Effektivität und Effizienz auf die Justiz. Dies geschieht im Rahmen sog. Neuer Steuerungsmodelle (NSM) wie der Kosten-Leistungsrechnung, des Controllings, des Benchmarkings und der Budgetierung.

Das Prinzip scheint simpel zu sein: In Zeiten knapper Haushaltsmittel ist das Erkennen und Nutzen von Einsparpotentialen nicht nur gut für den Steuerzahler, sondern scheinbar auch für die Gerichte selbst - können sie doch die dadurch gewonnen Ressourcen zur oft eingeforderten Entlastung nutzen. Jedoch wird dabei das Entscheidende übersehen: Jede dieser betriebswissenschaftlichen Methoden setzt zunächst die Bestimmung des angestrebten »Produkts« voraus. Was für die Wirtschaft noch leicht ist, erscheint für den Sektor Justiz dagegen unmöglich. Denn messbar sind nur quantitative Kriterien wie Anzahl der erledigten Verfahren, Dauer der einzelnen Arbeitsschritte, etc. Dagegen können Qualitätsmerkmale wie die Einhaltung von Verfahrensvorschriften und die durch die Urteile erzielte Rechtssicherheit und Verfahrensgerechtigkeit nicht quantifiziert werden, sie bleiben in diesen Modellen schlicht unberücksichtigt. ${ }^{17}$ Schneidet man diese wichtigere qualitative Dimension der gerichtlichen $\gg$ Dienstleistung « ab, begrenzt man auch das Prinzip der richterlichen Unabhängigkeit. Denn diese beinhaltet selbstredend den Aspekt der Handlungsfreiheit, welche es dem einzelnen Richter überlässt, die Sorgfalt und die Zeit für seine Arbeit selbst zu bestimmen. ${ }^{18}$ Das Festlegen zeitlicher Vorgaben, das Anhalten zur Verwendung von Textbausteinen, die Datenerhebung durch die Justizverwaltung bei den jeweiligen Richtern, all dies sind schwere Eingriffe in die richterliche Unabhängigkeit. ${ }^{19}$ Diese werden auch nicht dadurch abgemildert, dass man die Person des Festlegenden und Kontrollierenden durch andere Richter bestimmt. Denn für den betroffenen Richter ändert sich nichts - ob die Fremdbestimmung nun durch Vorgaben der Exekutive oder durch berufene Kontrollrichter erfolgt.

3. Abkoppelung der Justiz von rechtsstaatlichen Topoi und Neujustierung von Verfahrenzielen

Die Dritte Gewalt steht also inmitten administrativer Effizienzüberlegungen. Die Unabhängigkeit der Gerichte muss zu einer Abhängigkeit von der Ministerialbürokratie führen, ${ }^{20}$ wenn organisatorische Defizite, verursacht durch oftmals groteske Personalschlüssel, herrschen und wenn die Ressourcen der Landesjustizverwaltungen auf die treibenden Kräfte der politisch gewollten Präventionsstrategie - Polizei und Staatsanwaltschaft - verteilt werden.

17 Böttcher, Die Produkte der Justiz, in: Schulze-Fielitz (Hrsg.), Justiz und Justizverwaltung zwischen Ökonomisierungsdruck und Unabhängigkeit, Die Verwaltung Beiheft 5, 2002, 22.

18 Bertram/Daum/Graf von Schlieffen/Wagner, Das Neue Steuerungsmodell am Verwaltungsgerichtshof Hamburg, Möglichkeiten und Grenzen (Abschlussbericht der Arbeitsgruppe II), 1998, 102.

19 Stellungnahme der NRV vom 31.01.2008 zu ausgewählten Fragen des »Questionnaire for the 2008 CCJE opinion concerning the quality of judicial decisions«, 1ff., http://www.nrvnet.de/downloads_stellung/60.pdf.

20 Macke, Die Dritte Gewalt als Beute der Exekutive, in: DRiZ 1999, 481ff. 
a) Präventionswettbewerb

Auch der kriminalpolitische intendierte Präventionswettbewerb bedeutet die Auseinandersetzung innerhalb des Kriminaljustizsystems um knapper werdende Haushaltsmittel. Nur derjenige kann Ressourcen beanspruchen, der sich zweckrational als tauglich erweist, die herrschenden Politikmodelle in die Justizpraxis umzusetzen. Damit verkümmert die Dritte Gewalt zum bürokratischen Erfüllungsgehilfen, lässt sie im Verteilungskampf öffentlicher Mittel hintan stehen. Ihre Institutionen sind in der großen Gefahr, sich gegenüber den menschlichen Bedürfnissen und individuellen Freiheitsrechten des Bürgers zu verselbständigen. Auf Dauer muss dieser Prozess den demokratischen Rechtsstaat selbst destabilisieren. Den Kronzeugen de lege ferenda als Medium der »Präventionshilfe « zu bezeichnen ${ }^{21}$, belegt genau jene pseudologische Legitimationsstrategie, auch die - ursprünglich unabhängige - Justiz im Präventionswettbewerb erfolgreich aufzustellen.

b) Tendenz zu Geheimhaltung

Die Dominanz des exekutivischen Rechts zeigt sich auch in der kontinuierlichen Erweiterung operativer Ermittlungsbefugnisse. ${ }^{22}$ Deren Anwendung beeinflusst das Hauptverfahren und damit die Rolle der Strafjustiz nachhaltig. Die Tendenz der Geheimhaltung lässt die Ermittlungsbehörden den Beweisstoff so beeinflussen, dass den Gerichten wesentliche Erkenntnismöglichkeiten entzogen werden. In diesen der operativen Ermittlungsmethode der verdeckten Ermittlung geschuldeten Prüfungsprämissen sind die Gerichte gezwungen, den Weg ökonomischer Kalkulation zu beschreiten und sich darüber hinaus dem überhöhten Strafverfolgungsinteresse der Exekutive zu beugen. Genereller: Nicht nur die verdeckte Ermittlung, sondern auch die anderen auf Geheimhaltung gestützten operativen Ermittlungsmethoden konfrontieren die Gerichte mit Zumutungen an eine rechtsstaatliche Strafprozessdogmatik. ${ }^{23}$ Der täuschende, strafverfolgende Staat setzt die Maßstäbe und bestimmt die Lehre von den Beweisverboten. Operative Ermittlungsmethoden ziehen auch die Entformalisierung des gesamten nachfolgenden Strafverfahrens nach sich. Die Gerichte werden zu Subjekt und Gegenstand dieser Entformalisierung.

c) Formalisierte Richtervorbehalte

Es gehört zu den zweifelhaften Kompromissen aktueller Kriminalpolitik, nachhaltige Eingriffe in bürgerliche Freiheitsrechte mittels richterlicher Kontrollbefugnisse in Gestalt des Richtervorbehalts zu kompensieren. ${ }^{24}$ Normativ soll der Eindruck vermittelt werden, dass die mit der Erweiterung operativer Ermittlungsbefugnisse verbundene

21 Vgl. BT-Drs. 16/6268 (Entwurf eines Gesetzes zur Änderung des Strafgesetzbuches - Strafzumessung bei Aufklärungs- und Präventionshilfe).

22 Schünemann, Die Zukunft des Strafverfahrens - Abschied vom Rechtsstaat?, in: ZStW 119 (2007), 948ff.

23 Weßlau, Waffengleichheit mit dem Organisierten Verbrechen, KritV 1997, 238 ff.; Klawitter, Die Reduktion der Verteidigerrechte im Anti-OK-Sonderrechtssystem, KritV 1997, $248 \mathrm{ff}$.

24 BVerfGE 103, 142 (151); Asbrock, Zum Mythos des Richtervorbehalts, KritV 1997, 255. 
Ausdehnung exekutivischer Macht richterlich noch zu kontrollieren sei und dass die das Strafverfahren prägende Gewaltenbalance nicht tangiert werde. Der Richter wird zur letzten Hoffnung im Hinblick auf die Verteidigung von Grundrechten. Dieser bereits unsichere normative Anspruch wird durch die Rechtswirklichkeit des Richtervorbehalts in der strafprozessualen Praxis konterkariert. Im Ergebnis stellt der Richtervorbehalt ein dünnes rechtsstaatliches Feigenblatt für verfassungsrechtlich bedenkliche polizeiliche und staatsanwaltschaftliche Ermittlungsbefugnisse dar. ${ }^{25}$

d) Ökonomisierte Verfahrensziele und -methoden

Dem Vorwurf der Ineffizienz und übersteigerten Kosten von Rechtsstaatlichkeit des Strafverfahrens folgt die aktuelle kriminalpolitische Ausformung eines an der Kommunikationstheorie pseudologisch ankoppelnden Konsensprinzips.

\section{(1) Konsensualisierung}

Die deutsche Strafprozessordnung kennt bereits heute Verfahrensweisen, die flexibel und vermeintlich effizient, dafür aber wenig gerecht sind. Hierzu zählen die zahlenmäßig schon überwiegende Einstellung aus Opportunitätsgründen ( $§ 153 \mathrm{ff}$. StPO) sowie die Regelungen zum Strafbefehlsverfahren ( $\S 407 f f$. StPO). Diesen liegt ein konsensuales Verfahrensmodell zu Grunde, welches als Legitimation nicht mehr die materielle Wahrheit, sondern den Konsens vorsieht. Nicht mehr die formalisierte Suche nach der Wahrheit und die richterliche Aufklärungspflicht stehen im Vordergrund, sondern die Suche nach einem scheinbaren, in Wahrheit aber ökonomisch motivierten Konsens, weil schnelle Erledigung z.B. komplizierter Großverfahren möglich wird. ${ }^{26}$ Die richterliche Unabhängigkeit verliert nach diesem Modell ihre schützende Funktion. Eine Verurteilung setzt nicht mehr den positiven Nachweis der Schuld des Täters voraus, sondern lediglich dessen Einwilligung in die Rechtsfolge. Das Hauptverfahren wird zur Verhandlungsrunde. Der unbefangene und unparteiliche Richter wird zur gesprächsleitenden Ausgleichsperson degradiert. Der Wandel im richterlichen Rollenverständnis ist damit vollzogen. Der öffentliche Strafanspruch verkommt zur Verhandlungsmasse der Beteiligten.

Der Versuch des Ausbaus von Kommunikations- und Transparenzelementen verkennt die fundamentalen Prinzipien eines - auch europäischen - Strafrechts einer freiheitlichen Strafgesetzlichkeit. Der vorliegende Referentenentwurf des Bundesministeriums der Justiz ${ }^{27}$ und der Gesetzentwurf des Bundesrates ${ }^{28}$ sind ein weiterer Beleg des Abschleifens der erodierenden europäischen Strafrechtsprinzipien. Die Entwürfe verstehen sich zwar nur als normative Festschreibung der verfassungsrechtlichen und höchstrichterlichen Rechtsprechung, in dem das legalisiert werden soll, was angeblich bislang ohnehin schon praktiziert wird. Auch sollen Auswüchse und problematische Praktiken, z.B. bei fehlgeschlagenen Absprachen, dadurch vermieden werden. Unbe-

25 Backes/Gusy, Wer kontrolliert die Telefonüberwachung, 2003, $123 \mathrm{ff}$.

26 Trüg, Lösungskonvergenzen trotz Systemdivergenzen im deutschen und US-amerikanischen Strafverfahren, 2003, 112 m.w.N.

27 http://www.bmj.bund.de/files/-/1234/RefE\%20Verständigung.pdf.

28 BT-Drs. 16/4197. 
rücksichtigt bleibt dabei, dass das Leitprinzip des bisherigen Strafprozesses die materielle Wahrheitsaufklärung ist und das deutsche Strafprozessrecht bislang grundsätzlich vergleichsfeindlich ausgestaltet ist und kein Aushandeln von Schuld und Strafe im Sinne eines adversatorischen Parteiprozesses, in dem die Verfahrensbeteiligten über Beweise und Prozessverlauf disponieren, kennt. ${ }^{29}$

Das kontinentale Strafprozessrecht ist kontradiktorisch angelegt und wird durch gewichtige Prinzipien dauerhaft eingerahmt: Offizialprinzip, Akkusationsprinzip, Richtervorbehalt, Grundsatz des gesetzlichen Richters, Amtsermittlungsgrundsatz, Grundsatz rechtlichen Gehörs, Unmittelbarkeit, Mündlichkeit, Öffentlichkeit, Grundsatz der freien richterlichen Beweiswürdigung, Unschuldsvermutung, nemo-tenetur-Prinzip, Grundsatz des fairen Verfahrens, Gleichheitsgrundsatz und Grundsatz des schuldangemessenen Strafens. ${ }^{30}$ Damit unterliegt das Verfahrensergebnis prinzipiell nicht der Dispositionsfreiheit der Prozessbeteiligten. Es widerspricht kontinentalen Prozessauffassungen, den Beschuldigten als »vorleistungspflichtig « anzusehen. Im Prozess muss kein gegenseitiges Vertrauen hergestellt werden, sondern die staatliche Strafverfolgung hat Tat und Schuldbeweise strengbeweislich in den Prozess einzubringen. Das Recht, die erweiterte Kommunikation »im neuen Strafprozess zu initiieren, kann nur und ausschließlich beim Angeklagten liegen. Sonst gäbe es kein Halten mehr « mahnt der erfahrene Strafverteidiger Franz Salditt. Der Angeklagte hat in einem derartigen Konsensualverfahren keinen Anspruch mehr auf »rechtliches Gehör«, sondern der Richter muss den Angeklagten zum Gehör vermitteln und dann dessen Entscheidung erwarten. Dabei nehmen die Richter - so Salditt - »psychologisch eine den Angeklagten beratende Stellung ein $\ll{ }^{31}$ Das moderne Leitbild des Richters entwickelt sich damit zu einem Moderator, der auf »geschickte Weise « konsensuale Erledigung herbeiführt und Ressourcen einspart.

Richter kritisieren das als einen »Verfassungsverstoß der Landesregierungen«, denn diese begrenzten und reduzierten in ihren Haushaltsanträgen die Ressourcen für die Gerichte. Damit würden Strafrichter die Art und Weise der Rechtsanwendung zumindest teilweise nicht mehr autonom bestimmen, sondern sich durch Erledigungsdruck zum Dealen bestimmen lassen: »Dieser Zustand ist verfassungswidrig «. ${ }^{32}$ Verfassungskonforme Zustände können nur durch deutliche Ressourcenverstärkungen hergestellt werden, nicht hingegen durch die Abwälzung eines Gebots von Wohlwollen auf der Strafverfolgung unterworfene Bürger.

Im Übrigen: Die Dealstruktur zerstört auch die Redlichkeit im Strafverfahren. Der »Dumme«, der sogleich gesteht, hat nichts zu dealen. Die raffinierten Rechtsvertretungen sind die Gewähr des Zweiklassenstrafrechts nun von Gesetzes wegen. Ohnehin bleibt der Deal als dritte, auch in Zukunft informelle Möglichkeit neben dem formalisierten Verfahren erhalten. Salditt bezeichnet das als »Siegeszug des Freundstraf-

29 Trüg, Lösungskonvergenzen trotz Systemdivergenzen im deutschen und US-amerikanischen Strafverfahren, 2003, 145.

30 Vgl. dazu im Einzelnen Albrecht, Die vergessene Freiheit, 2. Auflage, 2006, 53ff.

31 Salditt, Kommunikation, in: StV 2007, 276.

32 Schulte-Kellinghaus, Zehn Thesen zu Absprachen (»Deals«) im Strafprozess, in: DRiZ 2007, 142. 
rechts«, das mit dem ständigen Anwachsen von Opportunitätseinstellungen ohnehin schon statistische Triumphe feiere. Verfahren durch Kommunikation und Konsens abzukürzen, wird als Partnerschaft auf Augenhöhe bezeichnet, womit Resozialisierung attestiert sei. Wer durch dieses Netz falle, dem sei nicht mehr zu helfen: »Die Ausgegrenzten gehören prozentual zur strafrechtlichen Unterschicht. « Während akademische Proteste gegen das Feindstrafrecht noch formiert würden, entwickele dieses sich zur Realität: »So gesehen, trägt das Reformkonzept zur Klärung der Klassifikation bei. Immerhin. ${ }^{33}$

Die richtige Diagnose aus dem Dilemma von ökonomischer Überlast, kriminalpolitisch-dogmatischer Unzulänglichkeit, normativer Überkommenheit und dem Allmachtsanspruch präventiver Übersteuerung gesellschaftlicher Probleme wäre ein Heckenschnitt überwuchernder Unzulänglichkeiten, das heißt juristisch übersetzt: normative Entkriminalisierung. ${ }^{34}$ Das wäre die notwendige Reduktion des Strafrechts auf scharf konturiertes Unrecht, welches in demokratischer Diskussion und unter Berücksichtigung europäischer Rechtskultur in öffentlicher Erörterung stattzufinden hätte. Zugleich wäre das ein Beitrag für eine echte europäische Integrationsbemühung, allein schon um drohenden europäischen Mehrheitsbeschlüssen, die nur auf der Basis eines minimalistischen Rechtsstaatsverständnisses zustande kommen, entgegenzuwirken.

\section{(2) Kronzeugenregelung}

Die geplante Kronzeugenregelung ist ein weiterer Mechanismus, der diesen Konsensforderungen entspringt. Der Staat will flexibel sein. Der öffentliche Strafanspruch und der Anspruch der Durchsetzung von Recht und Gesetz sind im Falle des Kronzeugenschutzes nicht selten sogar der richterlichen Überprüfung gänzlich entzogen. Ungleichbehandlungen und Rechtsunsicherheit finden so Eingang in das Strafverfahren. Der Tausch »Ermittlungs- oder Präventionshilfe « gegen Strafmilderung im Hauptverfahren ist ein schwerer Verstoß gegen das Schuldprinzip und verkehrt auch das Prinzip der Generalprävention in sein Gegenteil: Wer viele Verbrechen begeht, hat viel Verhandlungsmasse als Kronzeuge. Derartige »Aufklärungsmöglichkeiten« produzieren aber im kriminellen Milieu mehr Abschottung und Absicherung gegen Verrat. Der Zuwachs an Wahrheitsfindung wird überlagert durch eine Grundtendenz dauerhafter Fremdbezichtigung und führt zu Erschwernissen gerichtlicher Nachprüfung solcher vorteilsintendierter Aussagen. Wo darin eine Entlastung der Justiz liegen soll, ist unerfindlich. ${ }^{35}$

33 Salditt, Kommunikation, in: StV 2007, 276.

34 Kriminalpolitisch waren derartige Anregungen wissenschaftlicher Kommissionen zu Anfang der 90er Jahre noch gewünscht, was durch die beiden von den Landesregierungen in Hessen und Niedersachen eingesetzten rechtswissenschaftlichen Expertengruppen belegt wird: Albrecht/Hassemer/Voß (Hrsg.), Rechtsgüterschutz durch Entkriminalisierung, 1992; Albrecht/Beckmann/Frommel/Goy/Grünwald/Hannover/Holtfort/Ostendorf, Strafrecht ultima ratio, 1992. Die konkreten Empfehlungen normativer Entkriminalisierung fielen alsbald dem punitiven Populismus aller im Bundestag vertretenen Parteien zum Opfer.

35 Vgl. hierzu die Rede des Richters am Bundesgerichtshof a.D. und jetzigen Bundestagsabgeordneten Wolfgang Neskovic zum Thema »Kronzeugenregelung « vom 24.10.2007 im Deutschen Bundestag; Erstberatung zur Bundestagsdrucksache 16/6268 (abgedruckt in: KritV 4/ 2007), ebenso Mushoff, Die Renaissance der Kronzeugenregelung, in: KritV 2007, $366 f f$. 


\section{Die Randständigkeit der Dritten Gewalt im europäischen Kontext}

Was sich beim nationalen Richter vor Ort an Unabhängigkeitsverlust konkret abzeichnet, zeigt sich auch auf supra- und transnationaler Ebene an einer institutionellen Bedeutungslosigkeit der Dritten Gewalt. Europäische Einigung vollzieht sich derzeit primär in der Entwicklung einer starken, übermächtigen und kaum kontrollierten Exekutive. Die kriminalpolitische Konzentration der europäischen Rechtsentwicklung auf Polizei und Staatsanwaltschaften belegt das eindrucksvoll. Vor dem Hintergrund einer exekutivischen Informalisierung zum Zweck des Erreichens formaler europäischer Einheit liegt darin ein Verstoß gegen das europaweit geltende Prinzip der Gewaltenteilung. In einem Europa des Rechts müsste eine unabhängige Judikative parallel zu exekutivischen Institutionen entwickelt werden. Ein derartiger politischer Ansatz fehlt zurzeit gänzlich. Die europäische Exekutive schafft und kontrolliert sich selbst.

\section{Fehlende Kontrollfunktion der Dritten Gewalt}

Eine angemessene Überprüfbarkeit exekutiver Maßnahmen von EU-Organen fehlt sowohl auf europäischer als auch auf nationaler Ebene. So fehlt z.B. bei Europol der justizielle Überbau, eine gerichtliche Kontrolle ist nicht vorgesehen. Ferner ist auch den nationalen Gerichten die (strafgerichtliche) Rechtskontrolle wegen der Immunität aus Art. 41 EPÜ entzogen. Werden nationale Gerichte in den Vollzug europäischer Zwangsmaßnahmen eingebunden, ist die Wirksamkeit des gerichtlichen Rechtsschutzes eingeschränkt: Zum einen stehen tatsächliche Gründe der vollständigen Prüfung der Eingriffsvoraussetzungen entgegen (Sprachbarrieren, mangelnde Ressourcen etc.), zum anderen das Fehlen materieller Prüfungskompetenzen (z.B. im Rahmen der Vollstreckung des europäischen Haftbefehls). ${ }^{36}$ Dieses rechtsstaatliche Defizit macht deutlich, dass es an der normativen und institutionellen Konstruktion einer europäischen wirksamen Rechtskontrolle fehlt. Zur Zeit bestreitet lediglich die europäische Exekutive unangefochten das Feld der inneren Sicherheit.

Die Institution des Europäischen Gerichtshofes ist kein angemessenes judikatives Gegengewicht der Dritten Gewalt gegenüber Legislative und Exekutive. Der EuGH kontrolliert keine strafrechtliche Rechtsanwendung im Einzelfall, sondern - wenn überhaupt - Rechtsakte im Hinblick auf einzuhaltende Gesetzgebungszuständigkeiten auf europäischer Ebene. Im Übrigen: Die Konstruktion eines nationalen Richters vor Ort ist keine angemessene Kontrollmacht gegenüber der starken europäischen Exekutive. Der nationale Richter vor Ort gerät schon allein aufgrund der mangelnden sachlichen und personellen, sprachlichen und institutionellen Voraussetzungen in die Abhängigkeit des Zugriffs hochgerüsteter europäischer Strafverfolgung.

\section{Entleerung des Demokratieprinzips}

Rahmenbeschlüsse im Rahmen der noch ${ }^{37}$ existenten Dritten Säule sind Entscheidungen auf Ministerebene, also Entscheidungen der Exekutive. Anders als die vergemein-

36 Unger, Schutzlos ausgeliefert? Der europäische Haftbefehl, 2005.

37 Art. 69 e, f des Vertrages von Lissabon sieht die Abschaffung der Säulenarchitektur vor und überträgt strafrechtliche Regelungsbereiche in die Kompetenz supranationalen Rechts. Die damit zugleich implementierten Probleme dürften eine europäische Strafrechtsentwicklung noch problematischer machen (Vgl. zum Problem der »Notbremse« Art. 82 Abs. 3 bzw. Art. 83 Abs. 3 AEUV und hierzu Satzger in diesem Heft der KritV 1/2008). 
schafteten Normen der »Ersten Säule«, die mit ihrer Schaffung unmittelbare Verbindlichkeit für alle EU-Staaten entfalten, müssen Rechtsakte der »Dritten Säule« von den Einzelstaaten durch einen Umsetzungsakt in nationales Recht transformiert werden. Die Inhalte werden auf administrativer EU-Beamten-Ebene ausgehandelt, von Regierungen beschlossen und durch die staatlichen Parlamente nur noch exekutiert. Formal ist die Parlamentszustimmung zwar eine Legitimation durch Parlamentsbeschluss. Diese Formalie ist aber vor dem Hintergrund des weitreichenden Grundrechtsverzehrs der Bürger keine wirkliche Legitimation. Das Demokratieprinzip erfordert für legislative Akte, die im Prinzip untersagte nationale Kompetenzdelegationen betreffen, nicht nur eine formale sondern eine materielle Legitimation. Der Gesellschaftsvertrag erfordert, dass staatlicher Zwang nur durch Gesetze legitimiert ist, die sich die im Staat organisierten Bürger selbst gegeben haben.

Hieraus versteht sich das anspruchsvolle Programm partizipatorischer Demokratie. ${ }^{38}$ Dieses Programm wird durch die Attribute öffentlich und deliberativ gekennzeichnet. Öffentlich setzt danach eine öffentlich geführte, kritische und verständliche Diskussionsarena voraus, in der um den Gegenstand der Regelung heftig gestritten wird. Demokratie ist aus dieser Sicht ein primär öffentlicher Prozess. Deliberativ sei dieser Prozess, weil der Gesetzgeber Standards der Gesetzgebung einhalten muss: Beleg von Erfahrung, Beleg normativer Vertretbarkeit, Abschätzung von Folgewirkungen. Insgesamt ist mit diesem Kriterium die Möglichkeit öffentlicher Kontrolle institutioneller Macht verbunden. Zusammengefasst: Im Programm partizipatorischer Demokratie hat das Parlament die zentrale Aufgabe, Gesetzgebung aktiv zu gestalten - erst recht im Bereich der intergouvernementalen »Dritten Säule«. Das Gegenteil ist zurzeit der Fall. Bei Rahmenbeschlüssen versagt schon das Europäische Parlament als Kontrollorgan. Seine Beteiligung am Zustandekommen eines Rahmenbeschlusses erschöpft sich gemäß Art. 39 Abs. 1 EU in einem bloßen Anhörungsrecht. Hier ist nicht einmal ansatzweise parlamentarische Kontrolle und ein demokratisches Gegengewicht erkennbar.

Ist dieses Demokratiedefizit auf europäischer Ebene schon evident, zeigen sich verstärkende Defizite bei der Umsetzung in nationales Recht. Hier geht es nicht im Entferntesten um das »Ob«, sondern höchstens noch um das »Wie«. Der deutsche Gesetzgeber hat sich z.B. beim ersten Gesetz zum Europäischen Haftbefehl noch nicht einmal die möglichen Einschränkungen vorbehalten, die der Rahmenbeschluss selbst vorsieht (Einschränkung des Verzichts auf beiderseitige Strafbarkeit, Normierung restriktiver Gründe, aus denen die Vollstreckung des Europäischen Haftbefehls abgelehnt werden kann, Restriktionen der Rückwirkung). Der deutsche Gesetzgeber befindet sich also in einem zweifachen Dilemma: Ist der Gestaltungsspielraum schon durch die europäische und nationale Exekutive stark beschränkt, würde der notwendig exzessive Gebrauch parlamentarischer Kontroll- und Gestaltungsmöglichkeiten gegen die Umsetzungspflicht von Maßnahmen des Rates der EU verstoßen (vgl. Art. 34 Abs.

38 Höffe, Demokratie im Zeitalter der Globalisierung, 1999, S. 117 und Braum, Das Prinzip der gegenseitigen Anerkennung - Historische Grundlagen und Perspektiven europäischer Strafrechtsentwicklung, in: GA 2005, 681, 689. 
2 lit. b EU) - mithin politisch und rein äußerlich das europäische Einheitsstreben konterkarieren. Zum Beispiel wurde von Parlamentariern vor dem Bundesverfassungsgericht insofern nachvollziehbar beklagt, bei der Umsetzung des EU-Rahmenbeschlusses zum Europäischen Haftbefehl in »normativer Unfreiheit « gehandelt zu haben. ${ }^{39}$ Insgesamt kann man nach alledem mit den Kritikern in der Literatur die Missstände der parlamentarisch formellen Legitimation des Europäischen Haftbefehls als Entleerung des Demokratieprinzips bezeichnen. ${ }^{40}$

Der neue Reformvertrag löst die bisherige Säulenstruktur ab, er löst aber nicht die vorhandenen Kompetenzprobleme. Vorgesehen wird in einem neuen Titel I, der die Arten und Bereiche der Unionszuständigkeit definiert, dass die Union ausschließliche oder mit den Mitgliedsstaaten »geteilte Zuständigkeiten« hat. Der sog. »Raum der Freiheit, der Sicherheit und des Rechts« gehört zu dem Katalog »geteilter Zuständigkeiten«. Nach Art. 5 des Änderungsvertrages gelten zur Abgrenzung der Zuständigkeiten europäische Kernprinzipien, nämlich: begrenzte Ermächtigung, Subsidiarität und Verhältnismäßigkeit. Anhand dieser Prinzipien müsste der europäische Gesetzgeber begründen, dass ihm die Mitgliedsstaaten ein generelles Recht eingeräumt haben, das Strafrecht zu harmonisieren. Darüber hinaus müsste diese Strafrechtsharmonisierung nur auf europäischer Ebene, d.h. ohne die staatliche Souveränität zu berühren, möglich sein: Ein einheitliches Strafrecht in Europa ist damit auf absehbare Zeit Fiktion.

Was dagegen weiterhin möglich erscheint ist der punktuelle Zugriff europäischer Gesetzgebung auf das Strafrecht, nun auch gegebenenfalls nach den neuen Bestimmungen über eine »verstärkte Zusammenarbeit« (geregelt in Titel IV des EU-Reformvertrages). Denkbar sind danach Rechtsakte im Bereich des Strafrechts, an die nur die im Rahmen dieser Zusammenarbeit beteiligten Mitgliedsstaaten gebunden sind. Die fatale Perspektive wäre hierbei allerdings, dass die »EU der 27 « in mehrere Teilsysteme zersplittert, was der Einheitsorientierung der EU zuwiderliefe und einen normativen Flickenteppich in Europa zur Folge hätte.

\section{Unabhängigkeitserosion am Beispiel der Richterwahl (Amtszeit und Wiederwahl am EuGH und EGMR)}

Thesenhaft lässt sich formulieren, dass das Richterwahlverfahren intransparent, informell und parteipolitisch beeinflusst erscheint. Darüber hinaus liegen bei der Wiederwahl die Missbrauchsgefahr und parteipolitische Interessen auf der Hand. Europaweit enthält nur die österreichische Verfassung eine Regelung, wonach das Parlament bei der Auswahl von Richtern einzubeziehen ist. Damit entbehrt dieses europaweit gehandhabte Rekrutierungsverfahren weitestgehend einer hinreichenden demokratischen Legitimation. Das in Art. 223 EG geforderte »gegenseitige Einvernehmen« führt le-

39 Beleg bei Braum, Das Prinzip der gegenseitigen Anerkennung - Historische Grundlagen und Perspektiven europäischer Strafrechtsentwicklung, in: GA 2005, 681, 690 (Fn. 51).

40 Braum, wie Fn. 39; Unger, Schutzlos ausgeliefert? Der Europäische Haftbefehl - Ein Beispiel für die Missachtung europäischer Bürgerrechte, 2005, S. 79-89 und Schünemann, Europäischer Haftbefehl und EU-Verfassungsentwurf auf schiefer Ebene - Die Schranken des Grundgesetzes, in: ZRP 2003, 185, 186. 
diglich dazu, dass die anderen Mitgliedstaaten ein Vetorecht gegen die nationalen Entscheidungen haben: Davon wurde in der bisherigen Geschichte des Gerichtshofs noch nie Gebrauch gemacht.

Das Richteramt am EuGH erfordert nach sechs Jahren eine Bestätigungspflicht durch die Regierung (Art. 223 EG). Derartig kurze Amtzeiten - gerade mit der Möglichkeit der Wiederernennung - sind vor dem Hintergrund der Richterlichen Unabhängigkeit höchst problematisch. »Missliebige « Richter laufen so Gefahr, nicht erneut ernannt zu werden und verhalten sich deswegen möglicherweise erwartungs-, d.h. »regierungs-«gemäß. Hinzu kommt, dass Auswahl und Wahl allein durch exekutive Gremien - nämlich die nationalen Regierungen - erfolgen und sich dadurch faktisch einer öffentlichen Kontrolle entziehen ${ }^{41}$ Problematisch hieran sind vor allem die möglichen parteipolitischen Absprachen hinsichtlich der Bestätigungspraxis europäischer Richter. In der Literatur werden Beispiele hierfür benannt ${ }^{42}$ wobei selbst Oppositionsparteien dem in der Regel im Tausch für andere »EU-Posten « zustimmen. ${ }^{43}$ Deutlicher lässt sich richterliche Unabhängigkeit nicht konterkarieren.

Beim europäischen Gerichtshof für Menschenrechte sah es bislang nicht anders aus. Die Wiederwahl funktioniert nach dem gleichen Prinzip: jedes Land hat wiederum eine Liste mit drei Vorschlägen zu erstellen. Ein »faktischer Absetzungsbescheid«wie es der Schweizer Schubarth nennt - beinhaltet das Missbrauchspotential, einen »missliebigen« Richter nicht mehr auf diese Liste zu setzen. Dieses »Vetorecht« der Regierungen ${ }^{44}$ konterkariert die richterliche Unabhängigkeit, es hängt - wie das Schubarth anmerkt - »wie ein Damoklesschwert über den Richtern«; dies könne auch negative Auswirkungen auf die Bereitschaft haben, sich überhaupt als Kandidat zur Verfügung zu stellen. Der EGMR hat hieraus offensichtlich die richtige Konsequenz ziehen wollen. Im Europarats-Protokoll Nr. 14 zur EMRK vom 13. Mai 2004 ist die Wahl der Richter auf neun Jahre verlängert worden, ihre Wiederwahl soll in Zukunft nicht mehr zulässig sein. Bislang haben allerdings noch nicht alle Unterzeichnerstaaten dieses Protokoll ratifiziert.

Fast man die Situation der Dritten Gewalt auf europäischer Ebene zusammen, so ist kritisch festzustellen, dass es an einer gewalten-kontrollierenden Judikative im Bereich des Strafrechts fast vollständig mangelt. Man könnte zur Verdeutlichung überspitzt formulieren: Wo es keine richterliche Kontrolle gibt, können auch keine Erosionsprozesse stattfinden. Umso schwerwiegender sind die nachgewiesenen Beeinträchtigungen der Unabhängigkeit des Richters vor Ort in den einzelnen Mitgliedsländern. Im Übrigen zeigen sich bei den europäischen Gerichtshöfen schon vom Ansatz her zentrale Beschränkungen der Unabhängigkeit in Folge der kurzen Amtszeiten und der Wiederwahlkontrolle seitens der Exekutiven der Nationalstaaten.

41 Pieper, Verfassungsrichterwahlen, 1999, 64.

42 Siebert, Die Auswahl der Richter am Gerichtshof der Europäischen Gemeinschaften, 1997, 97.

43 Siebert a.a.O., 98.

44 Schubarth, Der europäische Richter - ein unabhängiger Richter?, in: FS Trechsel, 2002, 102. 


\section{Der Handlungsbedarf des nationalen und supranationalen Gesetzgebers im Hin- blick auf die Entstehung eines einheitlichen europäischen Strafrechts}

\section{Erste Forderung: Stärkung der Demokratie und Aktivierung parlamentarischer Kontrolle}

Aus dem neuen Grundlagenvertrag folgt, dass sich die Mitgliedstaaten - viel stärker als bisher - als Hüter des Subsidiaritätsprinzips verstehen müssen. Dies gilt insbesondere für die nationalen Parlamente, denen auch nach dem neuen Grundlagenvertrag eine wichtige Partizipationsfunktion im Rahmen europäischer (Straf-)-Gesetzgebung zukommt. Damit einher geht zugleich auch die Notwendigkeit, die Prinzipien des demokratischen Rechtstaats gegen usurpatorische kriminalpolitische Initiativen der EU zu verteidigen.

Das Beispiel des Europäischen Haftbefehls ist ein trauriger Beleg für eine von der Dritten Gewalt weitgehend unkontrollierte Kriminalpolitik der EU. Dieser Rahmenbeschluss wurde als Gegenstück zu den europäischen Grundfreiheiten konzipiert. Nicht maximale Freiheiten werden gegenseitig anerkannt, sondern maximale Freiheitsbeschränkungen. Wäre die Parallelität zur Warenverkehrsfreiheit und der dort geschaffenen Integrationsfreundlichkeit im Fokus europäischer Rechtspolitik, dann müsste das Prinzip lauten: Eine Handlung, die in einem Staat erlaubt ist, ist in allen Staaten erlaubt. Dann wäre aber ein Kernstrafrecht die Konsequenz, welches nicht nur beiderseitiger, sondern allseitiger Strafbarkeit entspräche. Der mit dem Prinzip gegenseitiger Anerkennung verknüpfte europaweite illiberale Effekt hat demgegenüber eine Nivellierung der Bürgerrechte auf das jeweils niedrigste Niveau im Querschnitt aller Mitgliedstaaten zur Folge. So ergibt sich ganz von selbst auf europäischer Ebene das Prinzip der maximalen Punitivität: Wenn man in einem Staat minimale Zeugnisverweigerungsrechte mit maximalen Abhörrechten der Polizei aus einem anderen Staat kombiniert, zerbricht die Gesamtbalance augenblicklich. ${ }^{45}$ Inwieweit ein derart durch gegenseitige Anerkennung produziertes europaweites maximal-punitives Verfahrensrecht europäische Integrationsleistungen bewirken könnte, bleibt unerfindlich.

\section{Zweite Forderung: Konstituierung justizförmiger Kontrolle der machtvollen euro- päischen Exekutive}

Der europäische Gesetzgeber schafft zwar außerhalb des Wirtschafts- und Umweltrechts nicht unmittelbar geltende europarechtliche Straftatbestände, sondern bedient sich - bislang - des Gesetzgebungsinstrumentes der Rahmenbeschlüsse, mittels dessen die jeweils schärfste Strafrechtspflege aller 27 Mitgliedstaaten zum Maßstab der europaweiten Rechtspraxis erhoben wird. Den 27 Kriminaljustizsystemen eine derart mit innerstaatlichen Rechtsprinzipien kollidierende Rechtspraxis zuzumuten, ist rechtsstaatlich schlechthin unerträglich.

45 Schünemann, Europäischer Haftbefehl und EU-Verfassungsentwurf auf schiefer Ebene Die Schranken des Grundgesetzes, in: ZRP 2003, 185, 186. 
Von einem gemeinsamen europäischen Strafrecht ist zurzeit nur ein illiberaler Effekt zu erwarten, und es bleibt beim derzeitigen Stand einer desolaten supranationalen Kriminalpolitik Aufgabe des nationalen Gesetzgebers, die europaweite Einebnung der Bürgerrechte für die Rechtsordnung der Nationalstaaten zu verhindern. Um künftig ähnlich schwere Verletzungen von Freiheitsrechten und um eine erneute Situation »normativer Unfreiheit« zu vermeiden, muss sich der parlamentarische Gesetzgeber zurückhalten, die EU zu weiterem Ausbau des Strafrechts zu ermutigen. Bis jetzt hat sich das Strafrecht in den Bereichen der Ökonomie und Umwelt durch die Hintertreppe in den europäischen Rechtsalltag eingeschlichen. Folgen sind Intransparenz der Gesetzgebung, eine diffuse Ausweitung der strafrechtlichen Zurechnung, unverständliche Normkonstruktionen, die weitgehend unkontrollierte Dominanz der Exekutive bei der Strafverfolgung und der Abbau rechtsstaatlicher Verfahrensprinzipien, ohne zugleich eine einheitliche justizförmige Kontrolle in Europa zu schaffen. Letztere muss vorab bestehen, bevor ein einheitliches europäisches Straf- und Strafverfahrensrecht europaweite Geltung beanspruchen kann. Das aber lässt sich nicht verordnen, nicht durch Mehrheitsbeschlüsse anordnen oder durch Rahmenbeschlüsse umsetzen.

\section{Dritte Forderung: Breite Förderung rechtspolitischer und wissenschaftlicher Initi- ativen zur Schaffung eines öffentlichen europäischen Rechtsbewusstseins}

Voraussetzung ist erst einmal die Schaffung eines öffentlichen europäischen Rechtsbewusstseins, das an den Prinzipien der europäischen Aufklärung ansetzen müsste. Hierzu bedürfte es einer breiten kriminalpolitischen Diskussion auf der offenen Bühne einer europäischen Rechtsstaatlichkeit und Öffentlichkeit. Diese müsste sich dazu bekennen, was überhaupt in Zukunft durch Strafrecht verfolgenswertes Unrecht zu sein hat. An dieser Debatte fehlt es zurzeit europaweit.

Einen positiven Ansatz bietet das Netzwerk europäischer Strafrechtler: European Criminal Law Academic Network (ECLAN). ${ }^{46}$ Diese neue Organisation führt Strafrechtler aus allen EU-Mitgliedstaaten zusammen, um prinzipiell und praktisch normative Bedingungen europäischer Strafrechtsentwicklung zu debattieren. Wichtiger Bestandteil der Arbeit dieses Netzwerks ist der Kontakt zu europäischen Institutionen, zu europäischen und nationalen Gerichten und zu nationalen Parlamenten. Diese Kontakte dienen einer qualitativen Bestandsaufnahme und Kritik europäischen Strafrechts. Es gehört zu den Aufgaben nationaler Parlamentarier, diese Debatte zur Kenntnis zu nehmen, sich an ihr zu beteiligen und Erkenntnisse daraus für jegliche Initiativen im Bereich europäischen Strafrechts zu verwerten. Die jüngst an der Universität Luxem-

46 Siehe dazu die von ECLAN organisierten wissenschaftlichen Tagungen und veröffentlichten Publikationen zur europäischen Strafrechtsentwicklung: Comment évaluer le droit pénal européen?, 2006, Weyembergh/De Biolley (Hrsg); in Deutschland ist ein Kreis um den Münchner Strafrechtslehrer Bernd Schünemann und Helmut Satzger auf europäischer rechtspolitischer Ebene aktiv; vgl. hierzu Schünemann (Hrsg.), Ein Gesamtkonzept für europäische Strafrechtspflege, 2006; vgl. auch die Beiträge dieses Heftes der KritV zur Anhörung des Unterausschusses Europa im Rechtsausschuss des Deutschen Bundestages am 28. November 2007 http://www.bundestag.de/ausschuesse/a06/anhoerungen/26_EU_Strafrecht/03_Stellungnahmen/index.html. 
burg unter Teilnahme zahlreicher EU-Richter und Generalanwälte stattgefundene internationale Konferenz zum Thema europäischer Justizkontrolle im europäischen Strafrechtsraum zeigt eine konstruktive und neue Richtung an, die es zu entwickeln gilt. ${ }^{47}$

Überfällig erscheint die Errichtung einer demokratisch legitimierten Kommission auf EU-Ebene zur Entwicklung eines europäischen Strafrechts mit Unterkommissionen in den nationalen Bereichen. Wissenschaftliche und kriminalpolitische Experten wären aufgerufen, der Öffentlichkeit, den Administrationen und den Parlamenten Anregungen und Diskussionsvorlagen zu präsentieren, die Ausgangspositionen für eine europaweite Debatte zur Wahrung und Entwicklung europäischer Rechtsprinzipien und Leitlinien für Strafrechtsentwicklungen bieten könnten. ${ }^{48}$

\section{Vierte Forderung: Wahrung und Förderung europäischer Rechtsprinzipien als Maßstab für ein europäischen Kernstrafrecht}

Dem nationalen Gesetzgeber ist daher ein Perspektivwechsel anzuraten. Zurückhaltung in der Gesetzgebung und Neugier für eine aufgeklärte, kriminalpolitische europäische Perspektive könnten Ansätze eines demokratischen, reflexiven europäischen Strafrechts sein, das die Bühne durch die Vordertür öffentlicher Diskurse betritt. Europa blickt auf eine lange und überzeugende Debatte eines rechtsstaatlichen Strafrechts zurück. Das Kernstrafrecht europäischer Strafgesetzlichkeit gilt es aus dieser Tradition heraus zu entwickeln. Das kann nur ein Strafrecht sein, das

- von Systemschutz-Anforderungen (z.B. Wirtschaft, Umwelt) entlastet ist,

- von verzerrenden symbolischen Strafrechtsanforderungen befreit ist und

- nach Maßgabe des Prinzips der Gewaltenteilung ein rechtsstaatliches Kriminaljustizsystem ermöglicht.

Diese drei Anforderungen an ein rechtsstaatliches Strafrecht bieten Anlass genug, eine europaweite Diskussion um ein Kernstrafrecht der Strafgesetzlichkeit öffentlich zu führen. Dieses Kernstrafrecht gründet auf wenigen, aber unverzichtbaren und größtenteils abwägungsfesten Prinzipien, die es zu sichern gilt. Eingebettet in das Prinzip der Freiheit als Erbe europäischer Aufklärung geht es

- um das Prinzip der Strafgesetzlichkeit als Fundament der verfassten Freiheit,

- um das Schuldprinzip als Begrenzung für die Strafmacht,

- um das Prinzip der Verhältnismäßigkeit als rechtliche Schranke von Gewaltanwendung,

- um das Legalitätsprinzip als Willkürschranke,

- um das Offizialprinzip als Garant des öffentlichen Strafrechts und

- um das Prinzip des fairen Verfahrens als Fundament des Strafprozesses im freiheitlichen Rechtsstaat.

47 Vgl. zu dem Luxemburg-Symposium 8./9. November 2007 die Beiträge in diesem Heft der KritV 1/2008.

48 Diesen Vorschlag hat Verfasser bei der Anhörung im Unterausschuss Europarecht des Rechtsausschusses des Deutschen Bundestages am 28. November unterbreitet. 
Europa braucht vor dem Hintergrund zurückliegender deprimierender Erfahrungen ein Strafrecht der Strafgesetzlichkeit, auf das die europäische Aufklärung hinaus wollte: eng, präzise, gesetzesgebunden, freiheitssichernd und - vor allem - als Bestandteil einer europäischen Verfassung, die diesen Namen auch verdient. 